\title{
Prevalence, Severity, and Predictors of Poststroke Depression in a Prospective Cohort of Jordanian Patients
}

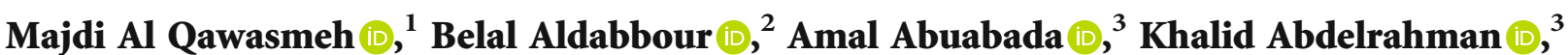 \\ Samah Elamassie $\mathbb{D}^{4},{ }^{4}$ Mays Khweileh ${ }^{(D)},{ }^{1}$ Mohammad Zahran ${ }^{D},{ }^{3}$ and Khalid El-Salem ${ }^{1}$ \\ ${ }^{1}$ Neurology Department, Faculty of Medicine, Jordan University of Science and Technology, Irbid, Jordan \\ ${ }^{2}$ Neuroscience Department, Faculty of Medicine, Islamic University of Gaza, P.O. Box 108, Gaza, State of Palestine \\ ${ }^{3}$ Psychiatry Department, Faculty of Medicine, Jordan University of Science and Technology, Irbid, Jordan \\ ${ }^{4}$ Health Services, United Nations Relief and Works Agency (UNRWA), Gaza, State of Palestine \\ Correspondence should be addressed to Belal Aldabbour; belal90md@gmail.com
}

Received 21 September 2021; Revised 15 December 2021; Accepted 20 December 2021; Published 7 January 2022

Academic Editor: Augusto Fusco

Copyright ( 2022 Majdi Al Qawasmeh et al. This is an open access article distributed under the Creative Commons Attribution License, which permits unrestricted use, distribution, and reproduction in any medium, provided the original work is properly cited.

\begin{abstract}
Poststroke depression (PSD) is common and remains a significant risk factor for poor outcomes. This prospective study is aimed at assessing the prevalence, severity, and predictors of PSD among Jordanian stroke survivors. A total of 151 patients who were consequently admitted to a tertiary teaching hospital with ischemic or hemorrhagic strokes were enrolled. Participants were screened on admission for premorbid depression using the PHQ-9 questionnaire; then, screening for PSD was repeated one and three months after stroke using the same tool. Depression prevalence at each screening was reported, and logistic regression analysis was conducted to evaluate for significant predictors. PHQ-9 scores suggestive of depression were reported by $15 \%, 24.83 \%$, and $17.39 \%$ of respondents on admission and after one and three months, respectively. Scores suggesting severe depression were reported by $0.71 \%, 2.13 \%$, and $6.52 \%$ of respondents, respectively. Significant predictors of PSD were having chronic kidney disease, current smoking status, moderate or severe disability (mRS score) at stroke onset, and severe dependence (BI) after one month ( $\boldsymbol{p}$ values $0.007,0,002,0.014$, and 0.031 , respectively). Patients with secondary and high school education levels were less likely to get depression compared with illiterate patients $(\boldsymbol{p} 0.042)$. This study showed that nearly one in four Jordanian stroke survivors experienced PSD after one month. In contrast, while the overall PSD prevalence declined towards the end of follow-up period, patients who remained depressed showed a tendency towards higher PSD severity.
\end{abstract}

\section{Introduction}

Stroke is a significant cause of morbidity and mortality globally [1]. It is the second leading noncommunicable cause of death in Jordan and a considerable source of complications and physical disability $[2,3]$. Stroke survivors are liable to a multitude of physical, psychiatric, social, and functional impacts. Poststroke depression (PSD) is the most frequent and a very important neuropsychiatric complication. Stroke survivors who develop PSD are at a greater risk of poor functional outcomes, lower quality of life, increasing cognitive impairment, recurrent vascular events, and higher mortality than those without depression [4-6].
The DSM-V defines poststroke mood disorders as mood disorders due to stroke, with the specifiers of depressive features, major depressive-like episode, or mixed mood features [6]. Studies evaluating PSD have reported varying findings owing to heterogeneous settings, populations, and methodologies. Also, several studies suggested that PSD prevalence differs with the time interval between stroke and depression assessment [7]. Additionally, some neurological symptoms such as aphasia and cognitive impairment may conceal mood abnormalities, and there is a lack of consensus over the best screening tool for case-finding $[7,8]$. Subsequently, the range of estimated PSD prevalence is wide $[9,10]$. However, meta-analyses with large databases reported that approximately one-third of survivors developed PSD at any 
time point up to 5 years following stroke [11-13]. Surveys from the Middle East and North Africa (MENA) region also reported a wide prevalence of PSD (17-73\%) [10], while previous surveys from Jordan reported a range from $25 \%$ to $76 \%$ [14-17]. Studies aiming to identify risk factors predisposing to PSD have also been inconsistent. The most frequently identified risk factors include the level of physical disability, stroke severity, and history of mental illness [7, 18].

Different instruments have been used to screen stroke survivors for PSD [19]. The Patient Health Questionnaire9 (PHQ-9) is a self-administered tool that employs nine standard questions to score each of the nine DSM criteria as zero (not at all) to three (nearly every day) [20]. It has been well-validated in different settings as a brief screener for PSD [19, 21, 22]. A meta-analysis found that PHQ-9 had a summary sensitivity of 0.77 and a specificity of 0.94 (0.90-0.97) [23]. Cut-off PHQ-9 scores of 5, 10, 15, and 20 represent mild, moderate, moderately severe, and severe depression, respectively [20]. This study is aimed at determining the prevalence and severity of PSD at one and three months after stroke and at investigating PSD predictors among Jordanian stroke survivors.

\section{Materials and Methods}

2.1. Study Design, Setting, and Ethical Considerations. This prospective cohort study was conducted at King Abdullah University Hospital (KAUH), the teaching hospital of Jordan University of Science and Technology (JUST) Faculty of Medicine. With over 680 beds, KAUH is the largest tertiary and teaching hospital in the north of Jordan. It offers emergency, clinic, and inpatient health care services to residents from the four northern Jordanian governorates and receives referrals from other hospitals within these governorates as well. Ethical approval for this study was obtained from the Institutional Review Board Committee at JUST (IRB approval number 279-2019). Written consent was obtained from patients upon enrolment, and oral consent was obtained from patients who were unable to write appropriately. Participants with positive PSD screening were informed about their results and offered psychiatric consultations.

2.2. Study Population and Case Identification. The study period extended over ten months from June 2019 to March 2020. It included all patients above sixteen who were admitted to the Neurology Department at KAUH with the primary diagnosis of a first or recurrent ischemic or hemorrhagic stroke. The study excluded patients who were younger than 16, patients with subarachnoid, subdural, or epidural hemorrhage, patients in whom the final diagnosis was transient ischemic attacks (TIA) or diagnoses other than acute stroke, patients with severe aphasia or advanced dementia precluding meaningful communication, and patients who refused to participate. Patients with recurrent strokes during the study period were recruited once only, and screening and follow-up were limited to the period preceding the second event.
2.3. Key Measures. Stroke was defined according to the 2013 AHA definition for ischemic and hemorrhagic strokes [24]. Stroke symptoms were reported as motor, sensory, dysarthria, aphasia, and others. Stroke severity was assessed using NIHSS score, with stroke severity stratified as mild (NIHSS less than 6), moderate (NIHSS 6-15), severe (16-25), and very severe (more than 25) [2, 25]. Disability was assessed using the modified Rankin Score (mRS), and a favorable outcome was identified as having $\mathrm{mRS}$ score of 2 or less [2, 25]. Functional status was assessed with Barthel Index (BI) [25], with the level of dependence classified as total (scores 0-20), severe (21-60), moderate (61-90), slight (91-99), and complete independence (100) [26]. Depression screening was conducted using the validated Arabic version of the PHQ-9 scale $[27,28]$, with cut-off scores of $5,10,15$, and 20 representing mild, moderate, moderately severe, and severe depression, respectively [20]. Hypertension, diabetes mellitus, and the other risk factors were defined according to standard guidelines, ascertained from previous notes or medical reports, or diagnosed by being on treatment for these conditions. Prolonged length of hospital stay (LOS) was defined as hospitalization lasting longer than the 75th percentile for the cohort [2].

2.4. Data Collection. Demographic data included age, gender, and education level. Each patient's final diagnosis, stroke severity on admission and discharge, stroke type, and presentation deficits were documented, as well as each patient's LOS and length of ICU stay. Stroke and PSD risk factors were ascertained, including hypertension, diabetes mellitus, heart failure, ischemic heart disease, cardiac arrhythmia, chronic kidney disease, previous ischemic or hemorrhagic stroke, smoking, and current or past history of psychiatric illness. Prestroke disability (mRS) and functional status (BI) were documented on admission and reassessed at discharge and after one month. As for depression screening, patients were screened on admission for premorbid depression, and screening was repeated via phone interviews conducted one and three months after discharge. In the last phone call, patients were asked if they had been to a psychiatrist or started on antidepressant medications after the stroke.

2.5. Statistical Analysis. Sample demographics and descriptive statistics were done first, reported as frequencies and proportions for categorical variables, and as mean (standard deviation "SD") and median (interquartile range "IQR") for continuous data. Chi-square and $\boldsymbol{t}$-test were used to assess the relationship between categorical variables and means, respectively. Logistic regression analysis was done to investigate the significant predictors of depression one month after stroke. Independent variables included age, gender, level of dependence after one month, education status, level of disability on admission, length of ICU admission, active smoking status, prior mood symptoms, diabetes mellitus, hypertension, ischemic heart disease, atrial fibrillation, chronic kidney disease, and heart failure. All statistical analyses were run in the SPSS software. The significance level $\alpha$ was established at 0.05 . 


\section{Results}

A total of 177 patients met the initial inclusion criteria, of which eight died within one month of the stroke, 11 were excluded due to severe stroke with persistent aphasia or impaired level of consciousness, two were excluded due to advanced dementia, and one due to language barriers precluding meaningful communication. Additionally, four patients dropped out of the study. Ultimately, 151 stroke survivors were included in the final analysis, of which 123 $(81.45 \%)$ responded to all three screenings, while the remaining 28 (18.54\%) missed either the first or the second follow-up screening after discharge. Figure 1 demonstrates study flow chart.

\subsection{Cohort Demographics, Risk Factor Profile, and Stroke} Severity. Males constituted $66.23 \%$ of the cohort (100 patients) and females $33.77 \%$ (51 patients). The mean age of the final cohort was 62.74 years (SD 12.42). The mean age was 60.82 (SD 12.63) for males and 66.902 (SD 11.04) for females. The difference between the two means was statistically significant ( $p$ 0.004). A low level of education was notable among females as $34(68 \%)$ were illiterate. On the other hand, 55 (55\%) of male patients held a diploma, a university degree, or higher education, and $88.89 \%$ had any level of education. Most (135 patients, $89.40 \%$ ) had at least one risk factor or comorbid medical condition before the stroke, with an overall mean of 2.377 (SD 1.43) comorbidities per patient. Hypertension was the most prevalent stroke risk factor (116, 76.82\%), followed by diabetes mellitus (91, $60.26 \%)$ and previous stroke (48, 31.79\%). Six (4.14\%) patients reported a history of psychiatric comorbidity. Smoking was more prevalent among male patients as 51 (51\%) were current smokers and $22(22 \%)$ were ex-smokers, while $76.46 \%$ of female patients were lifetime nonsmokers. Table 1 further illustrates these cohort demographics.

Most patients $(142,94 \%)$ suffered from an ischemic stroke, while $9(6 \%)$ had an intracerebral hemorrhage (ICH). The most frequently reported neurological symptom was motor deficits (106 patients, $70.20 \%$ ), followed by dysarthria (102 patients, $67.55 \%)$ and sensory deficits (50 patients, $33.11 \%$ ). The mean admission NIHSS score was 5.79 (SD 5.08), with a mean NIHSS of 5.59 (SD 4.79) among male patients and 6.20 (SD 5.65) among females. Also, most patients had a favorable prestroke disability status as the mean prestroke mRS score for the entire cohort was 0.424 (SD 1.15), and 139 (92.05\%) participants had a prestroke $\mathrm{mRS}$ score of two or less. The mean $\mathrm{mRS}$ score on discharge was 2.493 (SD 1.77), with $77(51.33 \%)$ patients having a favorable discharge mRS score ( 2 or less). The number of patients with a favorable mRS increased to $97(66.44 \%)$ one month after discharge. The mean discharge BI score was 68.66 (SD 33.84), and one month after discharge, it increased to 82.77 (SD 26.18). Mean LOS was 5.55 days (SD 5.57), and the median was four days (IQR 2-7). Prolonged LOS was noted in $32(21.19 \%)$ participants. As for ICU admission, 46 (30.46\%) of participants were admitted for at least one day, with a mean ICU stay of 4.11 (SD

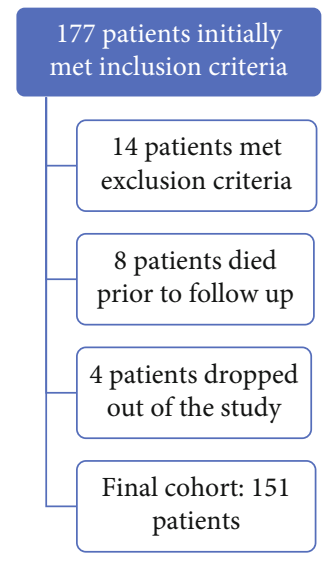

FIGURE 1: Study flowchart.

3.59) days. Tables 2 and 3 further demonstrate these characteristics.

3.2. Depression Prevalence and Severity. As Table 4 shows, 140 patients were screened on admission for premorbid depression. Of those, 21 (15\%) had positive screening. Scores suggestive of mild depression were reported by 15 patients (10.71\%), while moderate depression was reported by four patients $(2.85 \%)$. Scores suggesting moderately severe or severe depression were reported by one patient $(0.71 \%)$ for each category. Females were significantly more likely to be affected by premorbid depression (23.91\%) compared to males $(10.63 \%)$ (Chi-square statistic $=4.2687, p=0.039$ ). Screening for depression one month after admission revealed that 35 patients $(24.83 \%)$ reported any level of depression, representing an increase of $9.84 \%$ (Table 4 ). The percentage declined to $17.52 \%$ (24 patients) three months after admission, which was still higher than prestroke (admission) prevalence. Significantly, however, the severity of depression increased with time. Moderately severe and severe depression categories were reported by one patient each $(0.71 \%)$ on admission. In comparison, six (4.26\%) and seven (5.11\%) patients reported PHQ-9 scores suggestive of moderately severe depression one and three months after admission, while severe depression was reported by three $(2.13 \%)$ and nine $(6.57 \%)$ patients, respectively. In terms of treatment, only seven patients took antidepressant medications in the three months following the stroke. Moreover, five patients only had seen a psychiatrist during the same interval.

Finally, we looked at the PHQ-9 questionnaire to assess which domains were most likely to be affected in each screening (Table 5). On admission, the two questions that were most likely to get higher scores were the second question (feeling down, depressed, or hopeless) and the third question (trouble falling or staying asleep or sleeping too much), and the mean score was highest for the third question. One month after stroke, the second, third, and fourth questions were most likely to be affected, with the highest mean reported for the second question followed by the third question. Three months after stroke, the first and the second questions were the most likely to receive a positive answer 
TABLE 1: Cohort demographics and risk factor profile.

\begin{tabular}{|c|c|c|c|c|c|}
\hline Variables & Categories & $N 151$ & $\%$ & & \\
\hline \multirow{2}{*}{ Gender } & Male & 100 & 66.23 & Male (\%) N 100 & Female (\%) $N 51$ \\
\hline & Female & 51 & 33.77 & & \\
\hline \multirow{2}{*}{ Age } & 50 or less & 25 & 16.56 & $19(19.00)$ & $6(11.76)$ \\
\hline & Above 50 & 126 & 83.44 & $81(81.00)$ & $45(88.24)$ \\
\hline \multirow{5}{*}{ Education level* } & Illiterate & 45 & 30.21 & $11(11.11)$ & $34(68.00)$ \\
\hline & Primary school & 20 & 13.40 & $15(15.15)$ & $5(10.00)$ \\
\hline & Secondary and high school & 24 & 16.11 & $18(18.18)$ & $6(12.00)$ \\
\hline & Graduated and diploma & 51 & 34.23 & $46(46.46)$ & $5(10.00)$ \\
\hline & Higher education & 9 & 6.04 & $9(9.09)$ & $0(0.00)$ \\
\hline \multirow{4}{*}{ Smoking status } & Not a smoker & 66 & 43.71 & $27(27.00)$ & $39(76.47)$ \\
\hline & Smoker & 58 & 38.41 & $51(51.00)$ & $7(13.73)$ \\
\hline & Ex-smoker & 27 & 17.88 & $22(22.00)$ & $5(9.80)$ \\
\hline & Not a smoker and ex-smoker & 93 & $61.59 \%$ & $49(49.00)$ & $44(86.27)$ \\
\hline \multirow{9}{*}{ Comorbidities } & Hypertension & 116 & 76.82 & $71(71.00)$ & $45(88.24)$ \\
\hline & Diabetes & 91 & 60.26 & $58(58.00)$ & $33(64.71)$ \\
\hline & Previous stroke & 48 & 31.79 & $29(29.00)$ & $19(37.25)$ \\
\hline & Ischemic heart disease & 46 & 30.67 & $27(27.00)$ & $19(37.30)$ \\
\hline & Heart failure & 16 & 10.60 & $8(8.00)$ & $8(15.69)$ \\
\hline & Atrial fibrillation & 14 & 9.33 & $6(6.10)$ & $8(15.69)$ \\
\hline & Chronic kidney disease & 14 & 9.27 & $9(9.00)$ & $5(9.80)$ \\
\hline & Psychiatric comorbidity & 6 & 4.14 & $4(4.12)$ & $2(4.17)$ \\
\hline & Having other diseases & 32 & 21.19 & $18(18.00)$ & $14(27.45)$ \\
\hline \multirow{7}{*}{ Total number of comorbidities } & No comorbidities & 16 & 10.60 & $15(15.00)$ & $1(1.96)$ \\
\hline & One comorbidity & 23 & 15.23 & $16(16.00)$ & $7(13.73)$ \\
\hline & Two comorbidities & 46 & 30.46 & $29(29.00)$ & $17(33.33)$ \\
\hline & Three comorbidities & 35 & 23.18 & $26(26.00)$ & $9(17.65)$ \\
\hline & Four comorbidities & 20 & 13.25 & $9(9.00)$ & $11(21.57)$ \\
\hline & Five comorbidities & 7 & 4.64 & $4(4.00)$ & $3(5.88)$ \\
\hline & Six comorbidities & 4 & 2.65 & $1(1.00)$ & $3(5.88)$ \\
\hline
\end{tabular}

*Variability in totals is due to a missing value for one patient.

and recorded the highest means. Therefore, the most frequent complaint reported by patients at any interval was feeling down, depressed, or hopeless.

3.3. Predictors of Having PSD after One Month. Logistic regression analysis investigated the significant predictors of depression one month after stroke (Table 6). Among comorbidities, having chronic kidney disease increased the log odds of depression by $2.594(p=0.007)$. Active smoking status increased the log odds of depression by $2.187(p=0.002)$ versus nonsmokers and ex-smokers. Additionally, the binary logistic analysis revealed that patients with secondary and high school education levels were less likely to get depression than illiterate patients by $\log$ odd $-2.372(p=0.042)$. We also found that patients with total and severe dependence (by BI) one month after stroke were more likely to be depressed by $\log$ odds of 4.523 and 1.690 ( $p 0.039$ and $p 0.031$ ), respectively, compared with fully independent patients. Finally, patients with moderate or severe disability $(\mathrm{mRS}>2)$ on admission were more likely to be depressed by log odds of $2.311(p=0.014)$ in comparison to patients with milder or no disability early after the stroke. The remaining variables were not statically significant.

\section{Discussion}

This study assessed the prevalence and severity of PSD among Jordanian stroke survivors one and three months after admission, comparing the results with prestroke prevalence in the same group. Approximately one in four patients in our cohort experienced PSD one month after the stroke, and although the prevalence decreased at three months after the event, those who remained depressed had a worse PSD severity. We also identified several predictors for having PSD in this population, including initial disability status, functional performance after one month, level of education, active smoking, and having chronic kidney disease.

Previous local studies have reported varying figures. For instance, the first study evaluating PSD in Jordan was conducted in 2001 [14]. The authors defined PSD according to 
TABLE 2: Stroke type and severity.

\begin{tabular}{|c|c|c|c|c|c|}
\hline Variables & Categories & $N 151$ & $\%$ & Male (\%) N 100 & Female (\%) $N 51$ \\
\hline \multirow{2}{*}{ Type of stroke } & Ischemic & 142 & 94.00 & $95(95.00)$ & $47(92.16)$ \\
\hline & Hemorrhagic & 9 & 6.00 & $5(5.00)$ & $4(7.84)$ \\
\hline \multirow{4}{*}{ Length of hospital stay (LOS) } & 1-2 days & 38 & 25.17 & $24(24.00)$ & $14(27.45)$ \\
\hline & 3-4 days & 51 & 33.77 & $35(35.00)$ & $16(31.37)$ \\
\hline & 5-7 days & 30 & 19.87 & $20(20.00)$ & $10(19.61)$ \\
\hline & 7-42 days & 32 & 21.19 & $21(21.00)$ & $11(21.57)$ \\
\hline No ICU admission & & 105 & 70.47 & $72(73.47)$ & $33(64.71)$ \\
\hline \multirow{2}{*}{ Patients admitted to ICU } & $1-4$ & 27 & 61.36 & $17(65.38)$ & $10(55.56)$ \\
\hline & More than 4 & 17 & 38.64 & $9(34.62)$ & $8(44.44)$ \\
\hline \multirow{4}{*}{ Admission NIHSS scores } & Mild & 84 & 56.00 & $57(57.00)$ & $27(54.00)$ \\
\hline & Moderate & 57 & 38.00 & $38(38.00)$ & $19(38.00)$ \\
\hline & Severe & 8 & 5.33 & $5(5.00)$ & $3(6.00)$ \\
\hline & Very severe & 1 & 0.67 & $0(0.00)$ & $1(2.00)$ \\
\hline \multirow{10}{*}{ Presenting symptoms } & Motor deficit & 106 & 70.20 & $73(73.00)$ & $33(64.71)$ \\
\hline & Dysarthria & 102 & 67.55 & $69(69.00)$ & $33(64.71)$ \\
\hline & Sensory deficit & 50 & 33.11 & $32(32.00)$ & $18(35.29)$ \\
\hline & Dysphasia & 22 & 14.67 & $13(13.13)$ & $9(17.65)$ \\
\hline & Unsteadiness & 8 & 5.30 & $6(6.00)$ & $2(3.92)$ \\
\hline & Vision problem & 7 & 4.63 & $6(6.00)$ & $1(1.96)$ \\
\hline & Seizure & 4 & 2.65 & $3(3.00)$ & $1(1.96)$ \\
\hline & Vertigo & 3 & 1.99 & $2(2.00)$ & $1(1.96)$ \\
\hline & Dysphagia & 3 & 1.98 & $1(1.00)$ & $2(3.92)$ \\
\hline & Ataxia & 1 & 0.66 & $1(1.00)$ & $0(0.00)$ \\
\hline
\end{tabular}

TABle 3: Disability and functional status.

\begin{tabular}{|c|c|c|c|c|c|c|}
\hline \multirow{2}{*}{ Variables } & \multicolumn{2}{|c|}{ Before stroke } & \multicolumn{2}{|c|}{ At discharge } & \multicolumn{2}{|c|}{ At one month } \\
\hline & $N$ & $\%$ & $N$ & $\%$ & $N$ & $\%$ \\
\hline \multicolumn{7}{|c|}{ Modified Rankin score (dichotomous representation) } \\
\hline $0-2$ & 139 & 92.05 & 77 & 51.33 & 97 & 66.44 \\
\hline $3-5$ & 12 & 7.95 & 73 & 48.67 & 49 & 33.56 \\
\hline \multicolumn{7}{|l|}{ Modified Rankin score (detailed) } \\
\hline 0 no symptoms & 127 & 84.11 & 20 & 13.33 & 44 & 29.93 \\
\hline 1 no significant disability & 8 & 5.30 & 46 & 30.67 & 43 & 28.57 \\
\hline 2 slight disability & 4 & 2.65 & 11 & 7.33 & 11 & 7.48 \\
\hline 3 moderate disability & 4 & 2.65 & 7 & 4.67 & 15 & 10.20 \\
\hline 4 moderately severe disability & 4 & 2.65 & 45 & 30.00 & 27 & 18.73 \\
\hline 5 severe disability & 4 & 2.65 & 21 & 14.00 & 7 & 4.76 \\
\hline Mean (SD) & \multicolumn{2}{|c|}{$0.424(1.15)$} & \multicolumn{2}{|c|}{$2.493(1.77)$} & \multicolumn{2}{|c|}{$1.78(1.78)$} \\
\hline \multicolumn{7}{|l|}{ Barthel Index } \\
\hline Total dependence $(0-20)$ & - & - & 19 & 12.58 & 6 & 3.97 \\
\hline Severe dependence (21-60) & - & - & 42 & 27.81 & 22 & 14.57 \\
\hline Moderate dependence (61-90) & - & - & 22 & 14.57 & 32 & 21.19 \\
\hline Slight dependence (91-99) & - & - & 10 & 6.62 & 11 & 7.28 \\
\hline Full independence (100) & - & - & 58 & 38.41 & 80 & 52.98 \\
\hline Mean (SD) & \multicolumn{2}{|c|}{-} & \multicolumn{2}{|c|}{$68.66(33.84)$} & \multicolumn{2}{|c|}{$82.77(26.18)$} \\
\hline
\end{tabular}


TABle 4: Prevalence of depression.

\begin{tabular}{|c|c|c|c|c|c|}
\hline \multirow[b]{2}{*}{ Factors } & \multicolumn{5}{|c|}{ PHQ-9 score (depression severity) } \\
\hline & $\begin{array}{c}0-4 \text { (no } \\
\text { depression) } N \\
(\%)\end{array}$ & $\begin{array}{c}\text { 5-9 (mild } \\
\text { depression) } N(\%)\end{array}$ & $\begin{array}{l}\text { 10-14 (moderate } \\
\text { depression) } N(\%)\end{array}$ & $\begin{array}{c}\text { 15-19 (moderately severe) } \\
\text { depression } N(\%)\end{array}$ & $\begin{array}{c}20-27 \text { (severe } \\
\text { depression) } N(\%)\end{array}$ \\
\hline \multicolumn{6}{|c|}{ Depression screening on admission } \\
\hline $\begin{array}{l}\text { Total sample }(N \\
140)^{*}\end{array}$ & $119(85.00 \%)$ & $15(10.71 \%)$ & $4(2.86 \%)$ & $1(0.71 \%)$ & $1(0.71 \%)$ \\
\hline Males ( $N$ 94) & $84(89.36 \%)$ & $8(8.51 \%)$ & $1(1.06 \%)$ & $0(0.00 \%)$ & $1(1.06 \%)$ \\
\hline Females ( $N$ 46) & $35(76.09 \%)$ & $7(15.22 \%)$ & $3(6.52 \%)$ & $1(2.17 \%)$ & $0(0.00 \%)$ \\
\hline $\begin{array}{l}\text { Age above } 50(N \\
115)\end{array}$ & $97(84.35 \%)$ & $14(12.17 \%)$ & $3(2.61 \%)$ & $1(0.87 \%)$ & $0(0.00 \%)$ \\
\hline Age 50 or less ( $N 25)$ & $22(88.00 \%)$ & $1(4.00 \%)$ & $1(4.00 \%)$ & $0(0.00 \%)$ & $1(4.00 \%)$ \\
\hline Illiterate $(N 40)^{* *}$ & $32(80.00 \%)$ & $4(10.00 \%)$ & $3(7.50 \%)$ & $1(2.50 \%)$ & $0(0.00 \%)$ \\
\hline $\begin{array}{l}\text { Primary education } \\
(N \text { 16) }\end{array}$ & $13(76.47 \%)$ & $2(11.76 \%)$ & $1(5.88 \%)$ & $0(0.00 \%)$ & $1(5.88 \%)$ \\
\hline $\begin{array}{l}\text { Secondary or high } \\
\text { school }\left(\begin{array}{l}N \\
23\end{array}\right)\end{array}$ & $20(86.96 \%)$ & $3(13.04 \%)$ & $0(0.00 \%)$ & $0(0.00 \%)$ & $0(0.00 \%)$ \\
\hline Graduate (N 49) & $45(91.84 \%)$ & $4(8.16 \%)$ & $0(0.00 \%)$ & $0(0.00 \%)$ & $0(0.00 \%)$ \\
\hline Postgraduate ( $N$ 9) & $8(88.89 \%)$ & $1(11.11 \%)$ & $0(0.00 \%)$ & $0(0.00 \%)$ & $0(0.00 \%)$ \\
\hline \multicolumn{6}{|c|}{ Depression screening one month after stroke } \\
\hline Total sample ( $N$ 141) & $106(75.18 \%)$ & $13(9.22 \%)$ & $13(9.22 \%)$ & $6(4.26 \%)$ & $3(2.13 \%)$ \\
\hline Male ( $N$ 93) & $72(77.42 \%)$ & $10(10.75 \%)$ & $6(6.45 \%)$ & $3(3.23 \%)$ & $2(2.15 \%)$ \\
\hline Female $(N$ 48) & $34(70.83)$ & $3(6.25 \%)$ & $7(14.58 \%)$ & $3(6.25 \%)$ & $1(2.08 \%)$ \\
\hline $\begin{array}{l}\text { Age above } 50(N \\
117)\end{array}$ & $86(73.50 \%)$ & $11(9.40 \%)$ & $12(10.26 \%)$ & $6(5.13 \%)$ & $2(1.71 \%)$ \\
\hline Age 50 or less ( $N$ 24) & $20(83.33 \%)$ & $2(8.33 \%)$ & $1(4.17 \%)$ & $0(0.00 \%)$ & $1(4.17 \%)$ \\
\hline Illiterate $(N 41)$ & $29(70.73 \%)$ & $4(9.76 \%)$ & $6(14.63 \%)$ & $1(2.44 \%)$ & $1(2.44 \%)$ \\
\hline $\begin{array}{l}\text { Primary education } \\
(N \text { 17) }\end{array}$ & $10(58.82 \%)$ & $3(17.65 \%)$ & $2(11.76 \%)$ & $1(5.88 \%)$ & $1(5.88 \%)$ \\
\hline $\begin{array}{l}\text { Secondary or high } \\
\text { school }\left(\begin{array}{ll}N & 23\end{array}\right)\end{array}$ & $20(86.96 \%)$ & $0(0.00)$ & $3(13.04 \%)$ & $0(0.00)$ & $0(0.00)$ \\
\hline Graduate (N 49) & $38(77.55 \%)$ & $5(10.20 \%)$ & $2(4.08 \%)$ & $3(6.12 \%)$ & $1(2.04 \%)$ \\
\hline Postgraduate & $8(88.9 \%)$ & $1(11.11 \%)$ & $0(0.00 \%)$ & $0(0.00 \%)$ & $0(0.00 \%)$ \\
\hline \multicolumn{6}{|c|}{ Depression screening three months after stroke } \\
\hline Total sample ( $N$ 137) & $113(82.48 \%)$ & $2(1.46 \%)$ & $6(4.38 \%)$ & $7(5.11 \%)$ & $9(6.57 \%)$ \\
\hline Male ( $N$ 92) & $79(85.87 \%)$ & $0(0.00 \%)$ & $3(3.26 \%)$ & $5(5.43 \%)$ & $5(5.43 \%)$ \\
\hline Female $(N$ 46) & $35(76.09 \%)$ & $2(4.34 \%)$ & $3(6.52 \%)$ & $2(4.34 \%)$ & $4(8.70 \%)$ \\
\hline $\begin{array}{l}\text { Age above } 50(N \\
116)\end{array}$ & $96(82.76 \%)$ & $2(1.72 \%)$ & $4(3.45 \%)$ & $6(5.17 \%)$ & $8(6.90 \%)$ \\
\hline Age 50 or less $(N 22)$ & $18(81.82 \%)$ & $0(0.00 \%)$ & $2(9.09 \%)$ & $1(4.54 \%)$ & $1(4.54 \%)$ \\
\hline Illiterate ( $N$ 42) & $35(83.33 \%)$ & $1(2.38 \%)$ & $1(2.38 \%)$ & $2(4.76 \%)$ & $3(7.14 \%)$ \\
\hline $\begin{array}{l}\text { Primary education } \\
(N \text { 18) }\end{array}$ & $13(72.22 \%)$ & $1(5.56 \%)$ & $2(11.11 \%)$ & $0(0.00 \%)$ & $2(11.11 \%)$ \\
\hline $\begin{array}{l}\text { Secondary or high } \\
\text { school }\left(\begin{array}{l}N \\
22\end{array}\right)\end{array}$ & $18(81.82 \%)$ & $0(0.00 \%)$ & $2(9.09 \%)$ & $0(0.00 \%)$ & $2(9.09 \%)$ \\
\hline Graduate (N 47) & $40(85.10 \%)$ & $0(0.00 \%)$ & $1(2.13 \%)$ & $5(10.64 \%)$ & $1(2.13 \%)$ \\
\hline Postgraduate (N 7) & 7 (100.00\%) & $0(0.00 \%)$ & $0(0.00 \%)$ & $0(0.00 \%)$ & $0(0.00 \%)$ \\
\hline
\end{tabular}

${ }^{*} 11$ patients were not screened on admission due to severe stroke symptoms (e.g., dysarthria or aphasia). ${ }^{* *}$ Education data was missing for three patients.

the DSM-IV criteria for major depression and found that $25 \%$ of their cohort of 168 patients suffered from PSD three to four months after the event. They also reported that PSD was significantly more common among women survivors.
Another local study by Alghwiri in 2016 examined the relationship between PSD and physical well-being in a selected cohort of 61 Jordanian stroke survivors who were referred for physical therapy. It reported a PSD prevalence 
TABLE 5: The most affected domain at different screening periods.

\begin{tabular}{|c|c|c|c|c|c|c|}
\hline \multirow{2}{*}{$\begin{array}{l}\text { Screening time } \\
\text { Number and percentage of participants who answered } \\
\text { yes } \\
\text { and the mean score for all respondents }\end{array}$} & \multicolumn{2}{|c|}{ On admission } & \multicolumn{2}{|c|}{ After one month } & \multicolumn{2}{|c|}{ After three months } \\
\hline & $\begin{array}{l}N=140 \\
\quad(\%)\end{array}$ & Mean (SD) & $\begin{array}{l}N=141 \\
\quad(\%)\end{array}$ & Mean (SD) & $\begin{array}{l}N=26 \\
(\%)^{*}\end{array}$ & Mean (SD) \\
\hline \multicolumn{7}{|l|}{ Patient Health Questionnaire } \\
\hline Q1 Little interest or pleasure in doing things & $25(17.85)$ & $0.27(0.655)$ & $36(25.53)$ & $0.51(0.956)$ & $25(96.15)$ & $2.38(0.852)$ \\
\hline Q2 Feeling down, depressed, or hopeless & $33(23.57)$ & $0.33(0.662)$ & $41(29.08)$ & $0.59(1.011)$ & $25(96.15)$ & $2.23(0.951)$ \\
\hline $\begin{array}{l}\text { Q3 Trouble falling or staying asleep or sleeping } \\
\text { too much }\end{array}$ & $31(22.14)$ & $0.35(0.719)$ & $42(29.79)$ & $0.58(0.990)$ & $21(80.77)$ & $1.96(1.148)$ \\
\hline Q4 Feeling tired or having little energy & $23(16.36)$ & $0.26(0.650)$ & $42(29.79)$ & $0.52(0.893)$ & $24(92.31)$ & $2.08(1.017)$ \\
\hline Q5 Poor appetite or overeating & $20(14.28)$ & $0.24(0.645)$ & $27(19.15)$ & $0.37(0.834)$ & $16(61.54)$ & $1.27(1.185)$ \\
\hline $\begin{array}{l}\text { Q6 Feeling bad about yourself-or that you } \\
\text { are a failure or } \\
\text { have let yourself or your family down }\end{array}$ & $8(5.71)$ & $0.11(0.475)$ & $11(7.80)$ & $0.15(0.574)$ & $23(88.46)$ & $1.54(0.905)$ \\
\hline $\begin{array}{l}\text { Q7 Trouble concentrating on things, such as } \\
\text { reading the newspaper or watching television }\end{array}$ & $26(18.75)$ & $0.31(0.740)$ & $33(23.40)$ & $0.39(0.792)$ & $21(80.77)$ & $1.62(1.134)$ \\
\hline $\begin{array}{l}\text { Q8 Moving or speaking so slowly that other } \\
\text { people could } \\
\text { have noticed? Or so fidgety or restless that you have } \\
\text { been moving a lot more than usual }\end{array}$ & $6(4.28)$ & $0.07(0.372)$ & $12(8.51)$ & $0.14(0.503)$ & $20(76.92)$ & $1.81(1.167)$ \\
\hline $\begin{array}{l}\text { Q9 Thoughts that you would be better off dead, } \\
\text { or thoughts of hurting yourself in some way }\end{array}$ & $13(9.29)$ & $0.14(0.4383)$ & $15(10.64)$ & $0.20(0.659)$ & $18(69.23)$ & $1.19(1.021)$ \\
\hline
\end{tabular}

*The number represents patients who are depressed, who have been on an antidepressant medication, or both.

Table 6: Predictors of depression after one month.

\begin{tabular}{|c|c|c|c|c|}
\hline $\begin{array}{l}\text { Predictors of depression in the first month poststroke } \\
\text { Factors }\end{array}$ & $B$ & Wald & Sig. & $\operatorname{Exp}(B)$ \\
\hline Gender & 0.475 & 0.365 & 0.546 & 1.608 \\
\hline Age (reference less than 50 ) & -0.361 & 0.197 & 0.657 & 0.697 \\
\hline \multicolumn{5}{|l|}{ Barthel Index one month after the event } \\
\hline Total dependency $(0-20)$ & 4.523 & 4.246 & 0.039 & 92.145 \\
\hline Severe dependency (21-60) & 1.690 & 4.632 & 0.031 & 5.418 \\
\hline Moderate dependency (61-90) & 0.377 & 0.239 & 0.625 & 1.458 \\
\hline Slight dependency (91-99) & 1.139 & 1.527 & 0.217 & 3.123 \\
\hline \multicolumn{5}{|l|}{ Education level } \\
\hline Primary school & 0.483 & 0.326 & 0.568 & 1.621 \\
\hline Secondary and high school & -2.372 & 4.122 & 0.042 & 0.093 \\
\hline Graduated and diploma & -0.251 & 0.106 & 0.745 & 0.778 \\
\hline Postgraduate & -2.255 & 2.032 & 0.154 & 0.105 \\
\hline Modified ranking score on admission (reference $0-2$ ) & 2.311 & 6.029 & 0.014 & 10.087 \\
\hline \multicolumn{5}{|l|}{ Comorbidities and risk factors } \\
\hline Diabetes & -0.195 & 0.112 & 0.738 & 0.823 \\
\hline Hypertension & 0.699 & 0.800 & 0.371 & 2.011 \\
\hline Ischemic heart disease & -0.873 & 1.922 & 0.166 & 0.418 \\
\hline Atrial fibrillation & -1.442 & 1.072 & 0.301 & 0.237 \\
\hline Chronic kidney disease & 2.594 & 7.249 & 0.007 & 13.384 \\
\hline Heart failure & 0.454 & 0.180 & 0.672 & 1.575 \\
\hline Number of days the patients admitted to ICU & 0.174 & 1.784 & 0.182 & 1.190 \\
\hline Smoking (reference not smoker and ex-smoker) & 2.187 & 9.497 & 0.002 & 8.912 \\
\hline Prior mood symptoms & -1.951 & 2.037 & 0.154 & 0.142 \\
\hline
\end{tabular}


of $64 \%$ using the Beck Depression Inventory (PDI) questionnaire [16]. Similarly, Almhdawi et al. in 2020 evaluated 153 Jordanian stroke survivors with stroke chronicity of at least four months and who were receiving occupational therapy [17]. The investigators used the Depression Anxiety Stress Scale (DASS 21) and reported PSD in $74.5 \%$ of the cohort. However, both studies selected patients from physical or occupational therapy facilities, and participants had prominent physical disability and/or balance problems [16, 17]. Also, each of these studies had a different methodology and setting, and patients were screened at various intervals after stroke, which ultimately resulted in variable and generally noncomparable estimates of PSD prevalence.

Another cross-sectional study by Ayasrah et al. in 2017 evaluated PSD among 198 hospitalized patients with stroke in nine Jordanian hospitals using the Hospital Depression Subscale (HDS) of the Hospital Anxiety and Depression (HAD) scale [15]. The authors reported PSD in $51.6 \%$ of the sample. Yet again, different population characteristics, study settings, and screening tools may have predisposed for reporting such a high number. For instance, the mean age of patients was six years younger than our cohort, and $80.3 \%$ of the cohort were unable to perform activities of daily living without help (versus $40.39 \%$ of our cohort during admission). Also, patients were screened in a hospital setting, and the screening interval after the stroke was not standardized between patients. In addition, HDS has a lower positive predictive value (25-55\%) for the used cut-off score than PHQ-9 (46-69\%) [19, 29, 30], and the scale has one of the highest required literacy levels of the self-completed depression screening instruments [31].

On the other hand, three of the PSD risk factors identified by Ayasrah et al. correlated with our findings: having a lower level of education, being a smoker, and level of functional dependence after the stroke [15]. Physical disability and stroke severity also were significant predictors identified by Alghwiri in addition to cognitive impairment [16]. Therefore, our study's findings are consistent with previous literature and underline the considerable impact of stroke severity on the different outcome measures, including psychiatric complications [32].

Smoking was a significant predictor of PSD in both our study and the study by Ayasrah et al. [15]. It was also a significant predictor of PSD in other studies from our region and other regions $[33,34]$. It has been suggested that this correlation may be due to vitamin D deficiency in smokers in addition to the dysphoric effects of nicotine abstinence $[33,34]$. We suggest that it may also reflect feelings of guilt in stroke survivors with history of heavy smoking or in those who continue to smoke. This may be particularly relevant in Jordan, where the country is known to have one of the world's highest smoking rates [35] and where smoking has been linked to higher stroke severity after having a first ischemic stroke [2].

The natural history of poststroke depression is dynamic [36]. In this study, we found that the overall PSD prevalence was higher at the first month after stroke than prevalence at the third month, although severe PSD continued to increase with time. This finding likely reflects part of the natural his- tory of PSD among Jordanian patients up to three months after stroke. In addition, other studies that have assessed stroke survivors more than once suggested that most patients had PSD soon after the event, and a majority had recovered in subsequent assessments [12]. Moreover, very few patients in our cohort saw a psychiatrist or took an antidepressant medication, which could have impacted their PSD severity on longitudinal follow-up.

Understanding the pathophysiology of PSD may decrease the uncertainty about its treatment and prevention strategies [7]. Nonetheless, the mechanisms underlying PSD are not well-defined owing to the complexity of its pathways and the heterogeneity of clinical studies [7, 18]. Possible contributing factors include lesion location [37], reductions in neurogenesis and/or angiogenesis [18, 38], immune dysfunction particularly in the limbic area [39], neurotransmitter dysfunction [40,41], and hypothalamic-pituitary-adrenal (HPA) axis activation after stroke [42]. Moreover, it is believed that PSD is clinically underdiagnosed due to physician, patient, and caregiver under recognition $[7,18]$. This highlights the necessity of increasing awareness about PSD symptoms among medical practitioners and the general population and demonstrates the need for a standard screening tool for PSD.

This study is not without limitations. It was conducted in a single center and excluded patients with advanced dementia or severe aphasia. Also, lasting effects of the stroke itself, such as residual neglect and cognitive impairment, may impact patient awareness about their symptoms. Additionally, follow-up was limited to three months after the stroke. Finally, although PHQ-9 is a recognized screening tool for PSD, the gold standard for diagnosis remains through clinician-administered structured clinical interviews.

\section{Conclusions}

Approximately one in four Jordanian stroke survivors in our cohort experienced PSD one month after the stroke. Although prevalence at three months was lower, the severity of depression increased towards the end of follow-up. Significant PSD predictors included the initial disability status, functional performance after one month, level of education, active smoking, and having chronic kidney disease. This study demonstrates the need for better awareness about PSD among medical practitioners and the general population and underscores the global need for a standard screening tool for PSD.

\section{Data Availability}

The data is available from Belal Aldabbour upon reasonable request.

\section{Conflicts of Interest}

The authors declare that there is no conflict of interest regarding the publication of this paper. 


\section{References}

[1] M. Katan and A. Luft, "Global burden of stroke," Seminars in Neurology, vol. 38, no. 2, pp. 208-211, 2018.

[2] M. A. Qawasmeh, B. Aldabbour, A. Momani et al., "Epidemiology, Risk Factors, and Predictors of Disability in a Cohort of Jordanian Patients with the First Ischemic Stroke," Stroke Research and Treatment, vol. 2020, Article ID 1920583, 9 pages, 2020.

[3] R. W. Jaradat, A. B. Lahlouh, O. Y. Alshogran, B. A. Aldabbour, and A. A. Balusha, "Nosocomial infections among patients with intracra-nial hemorrhage: a retrospective data analysis of predictors and outcomes," Clinical Neurology and Neurosurgery, vol. 182, pp. 158-166, 2019.

[4] Q. E. Wu, A. M. Zhou, Y. P. Han et al., "Poststroke depression and risk of recurrent stroke," Medicine, vol. 98, no. 42, article e17235, 2019.

[5] F. Bartoli, C. Di Brita, C. Crocamo, M. Clerici, and G. Carra, "Early post-stroke depression and mortality: meta-analysis and me-ta-regression," Frontiers in Psychiatry, vol. 9, p. 530, 2018.

[6] R. G. Robinson and R. E. Jorge, "Post-stroke depression: a review," The American Journal of Psychiatry, vol. 173, no. 3, pp. 221-231, 2016.

[7] A. Towfighi, B. Ovbiagele, N. el Husseini et al., "Poststroke depression: a scientific state-ment for healthcare professionals from the American Heart Association/American Stroke Association," Stroke, vol. 48, no. 2, pp. e30-e43, 2017.

[8] N. Meader, T. Moe-Byrne, A. Llewellyn, and A. J. Mitchell, "Screening for poststroke major depression: a meta-analysis of diagnostic validity studies," Journal of Neurology, Neurosurgery, and Psychiatry, vol. 85, no. 2, pp. 198-206, 2014.

[9] Y. Shi, D. Yang, Y. Zeng, and W. Wu, "Risk factors for poststroke depression: a meta-analysis," Frontiers in Aging Neuroscience, vol. 9, p. 218, 2017.

[10] M. I. Kaadan and M. J. Larson, "Management of post-stroke depression in the Middle East and North Africa: too little is known," Journal of the Neurological Sciences, vol. 378, pp. 220-224, 2017.

[11] M. L. Hackett and K. Pickles, "Part I: frequency of depression after stroke: an updated systematic review and meta-analysis of ob-servational studies," International Journal of Stroke, vol. 9, no. 8, pp. 1017-1025, 2014.

[12] L. Ayerbe, S. Ayis, C. D. Wolfe, and A. G. Rudd, "Natural history, predictors and outcomes of depression after stroke: systematic re-view and meta-analysis," The British Journal of Psychiatry, vol. 202, no. 1, pp. 14-21, 2013.

[13] A. J. Mitchell, B. Sheth, J. Gill et al., "Prevalence and predictors of post-stroke mood disorders: a meta-analysis and metaregression of depression, anxiety and adjustment disorder," General Hospital Psychiatry, vol. 47, pp. 48-60, 2017.

[14] R. Bayer, Z. Marji, and W. Shunaigat, "Frequency and clinical determinants of major post stroke depression in Jordan," Qatar Medical Journal, vol. 10, pp. 47-50, 2001.

[15] S. M. Ayasrah, M. M. Ahmad, and I. A. Basheti, "Post-stroke depression in Jordan: prevalence correlates and predictors," Journal of Stroke and Cerebrovascular Diseases, vol. 27, no. 5, pp. 1134-1142, 2018.

[16] A. A. Alghwiri, "The correlation between depression, balance, and physical functioning post stroke," Journal of Stroke and Cerebrovascular Diseases, vol. 25, no. 2, pp. 475-479, 2016.
[17] K. A. Almhdawi, A. Alazrai, S. Kanaan et al., "Post-stroke depression, anxiety, and stress symptoms and their associated factors: a cross-sectional study," Neuropsychological Rehabilitation, vol. 31, no. 7, pp. 1091-1104, 2021.

[18] Y.-J. Lai and L. McCullough, "Poststroke Depression: Pathophysiology and Treatment Strategies," Neurobiology of Depression, pp. 197-205, 2019.

[19] L. J. Burton and S. Tyson, "Screening for mood disorders after stroke: a systematic review of psychometric properties and clinical utility," Psychological Medicine, vol. 45, no. 1, pp. 2949, 2015.

[20] K. Kroenke, R. L. Spitzer, and J. B. Williams, "The PHQ-9: validity of a brief depression severity measure," Journal of General Internal Medicine, vol. 16, no. 9, pp. 606-613, 2001.

[21] B. Levis, A. Benedetti, B. D. Thombs, and D. E. S. D. Collaboration, "Accuracy of Patient Health Questionnaire-9 (PHQ-9) for screening to detect major depression: individual participant data meta-analysis," BMJ, vol. 365, p. 1476, 2019.

[22] L. S. Williams, E. J. Brizendine, L. Plue et al., "Performance of the PHQ-9 as a screening tool for de-pression after stroke," Stroke, vol. 36, no. 3, pp. 635-638, 2005.

[23] K. A. Wittkampf, L. Naeije, A. H. Schene, J. Huyser, and H. C. van Weert, "Diagnostic accuracy of the mood module of the Patient Health Questionnaire: a systematic review," General Hospital Psychiatry, vol. 29, no. 5, pp. 388-395, 2007.

[24] R. L. Sacco, S. E. Kasner, J. P. Broderick et al., "An updated definition of stroke for the 21st Century," Stroke, vol. 44, no. 7, pp. 2064-2089, 2013.

[25] K. Ghandehari, "Challenging comparison of stroke scales," Journal of Research in Medical Sciences, vol. 18, no. 10, pp. 906-910, 2013.

[26] C. V. Granger, C. C. Sherwood, and D. S. Greer, "Functional status measures in a comprehensive stroke care program," Archives of Physical Medicine and Rehabilitation, vol. 58, no. 12, pp. 555-561, 1977.

[27] H. Belhadj, R. Jomli, U. Ouali, Y. Zgueb, and F. Nacef, "Validation of the Tunisian version of the patient health questionnaire (PHQ-9)," European Psychiatry, vol. 41, no. S1, p. S523-S, 2017.

[28] I. Pfizer, PHQ-9 Patient Depression Questionnaire. Arabic versionAugust 2021, http://www.phqscreeners.com/images/sites/ g/files/g10060481/f/201412/PHQ9_Arabic\%20for\%20Tunisia .pdf.

[29] P. Dajpratham, P. Pukrittayakamee, W. Atsariyasing, K. Wannarit, J. Boonhong, and K. Pongpirul, "The validity and reliability of the PHQ-9 in screening for post-stroke depression," BMC Psychiatry, vol. 20, no. 1, p. 291, 2020.

[30] J. R. Williams, E. S. Hirsch, K. Anderson et al., “A comparison of nine scales to detect depression in Parkinson disease: which scale to use?," Neurology, vol. 78, no. 13, pp. 998-1006, 2012.

[31] J. W. Williams Jr., M. Pignone, G. Ramirez, and S. C. Perez, "Identifying depression in primary care: a literature synthesis of case-finding instruments," General Hospital Psychiatry, vol. 24, no. 4, pp. 225-237, 2002.

[32] M. L. Hackett and C. S. Anderson, "Predictors of depression after stroke: a systematic review of observational studies," Stroke, vol. 36, no. 10, pp. 2296-2301, 2005.

[33] E. M. Khedr, A. A. Abdelrahman, T. Desoky, A. F. Zaki, and A. Gamea, "Post-stroke depression: frequency, risk factors, and impact on quality of life among 103 stroke 
patients-hospital-based study," The Egyptian Journal of Neurology, Psychiatry and Neuro-surgery, vol. 56, no. 1, p. 66, 2020.

[34] W. Ren, Y. Gu, L. Zhu et al., "The effect of cigarette smoking on vitamin $\mathrm{D}$ level and depression in male patients with acute ischemic stroke," Comprehensive Psychiatry, vol. 65, pp. 9-14, 2016.

[35] T. K. Burki, "Tobacco control in Jordan," The Lancet Respiratory Medicine, vol. 7, no. 5, p. 386, 2019.

[36] L. Ayerbe, S. Ayis, S. Crichton, C. D. Wolfe, and A. G. Rudd, "The natural history of depression up to 15 years after stroke: the South London Stroke Register," Stroke, vol. 44, no. 4, pp. 1105-1110, 2013.

[37] L. Terroni, E. Amaro, D. V. Iosifescu et al., "Stroke lesion in cortical neural circuits and post-stroke incidence of major depressive episode: a 4-month prospective study," The World Journal of Biological Psychiatry, vol. 12, no. 7, pp. 539-548, 2011.

[38] G. Masi and P. Brovedani, "The hippocampus, neurotrophic factors and depression: possible implications for the pharmacotherapy of depression," CNS Drugs, vol. 25, no. 11, pp. 913-931, 2011.

[39] G. Spalletta, L. Cravello, F. Imperiale et al., "Neuropsychiatric symptoms and interleukin-6 serum levels in acute stroke," The Journal of Neuropsychiatry and Clinical Neurosciences, vol. 25, no. 4, pp. 255-263, 2013.

[40] X. W. Ji, C. L. Wu, X. C. Wang, J. Liu, J. Z. Bi, and D. Y. Wang, "Monoamine neurotransmitters and fibroblast growth factor-2 in the brains of rats with post-stroke depression," Experimental and Therapeutic Medicine, vol. 8, no. 1, pp. 159-164, 2014.

[41] H. Q. Gao, H. Y. Zhu, Y. Q. Zhang, and L. X. Wang, "Reduction of cerebrospinal fluid and plasma serotonin in patients with post-stroke depression: a preliminary report," Clinical and Investigative Medicine, vol. 31, no. 6, pp. E351-E356, 2008.

[42] J. Weidenfeld, R. R. Leker, N. Gai, A. Teichner, D. Bener, and H. Ovadia, "The function of the adrenocortical axis in permanent middle cerebral artery occlusion: effect of glucocorticoids on the neurological outcome," Brain Research, vol. 1407, pp. 90-96, 2011. 